\title{
Spectral Distribution of Scattered Light from a Chemical Relaxation System
}

\author{
Wilson Marques Jr.*, Gilberto M. Kremer*, Miriam Pandolfi Bianchi ${ }^{\dagger}$ and \\ Ana Jacinta Soares** \\ *Departamento de Física, Universidade Federal do Paraná, Curitiba, Brazil \\ ${ }^{\dagger}$ Dipartimento di Matematica, Politecnico di Torino, Turin, Italy \\ ${ }^{* *}$ Departamento de Matemática e Aplicações, Universidade do Minho, Braga, Portugal
}

\begin{abstract}
The aim of this work is to describe the light scattering spectra of a quaternary reacting gas mixture from the macroscopic field equations derived from the kinetic BGK-type model proposed by the authors in a previous paper. The study is developed in a hydrodynamic regime for which the system of the field equations of constituent number densities, momentum and temperature of the mixture is closed by the constitutive equations for rate of reaction, diffusion velocities, pressure tensor and heat flux vector. The spontaneous Rayleigh-Brillouin scattering is calculated from the constituent density perturbations of the linearized field equations, and its line shape is drawn for two different mixtures of the Hydrogen-Chlorine system showing the induced chemical reaction effect.
\end{abstract}

Keywords: Boltzmann equation, Chemical reactions, Light scattering

PACS: $51.10 .+\mathrm{y}$, 47.70.Fw, 78.35.+c

\section{INTRODUCTION}

When light interacts with a gaseous reactive mixture at thermodynamic equilibrium, the corresponding spectrum of the scattered light gives back a lot of information on non-equilibrium thermodynamics and transport properties of the gas and, in particular, on the decay of the thermal density fluctuations which is mainly ruled by the transport coefficients. Many papers have been written on this matter within experimental and theoretical grounds (see e.g. the review article [1]). The present paper deals with a gas mixture of four constituents undergoing a chemical reaction of type $A+B \rightleftharpoons C+D$ described by the BGK-type model proposed by the authors in the previous paper [2]. Elastic cross sections of rigid spheres and reactive cross sections with activation energy are considered. In the hydrodynamic description of the mixture, the reactive balance laws for the basic fields of constituent number densities, mean velocity and temperature of the whole mixture are closed at the Navier-Stokes, Fourier and Fick level in a flow regime for which the chemical affinity is a small quantity. Accordingly, the transport coefficients of diffusion, shear viscosity and thermal conductivity are explicitly determined and the model is suitable to study the light scattering phenomenon. The main purpose consists in evaluating the spectral distribution of light scattered by thermal density fluctuations. The analysis here follows the hydrodynamic approach used in those research works (see e.g. paper [3]) for which the gas density fluctuations involve low frequencies and long wave length. The spectral distribution is directly related to the dynamic structure factor $S(q, \omega)$ which is explicitly computed, once the auto and cross correlation functions of the density fluctuations have been determined through the Fourier-Laplace transforms of the field perturbations predicted by the linearized hydrodynamic system of the assumed relaxation model.

\section{MODEL EQUATIONS}

At the aim of studying the light scattering phenomenon in the quaternary reactive mixture, an approximate kinetic model equation compatible with the BGK-type approach has been considered. The main idea underlying this model, first proposed in paper [2], is to replace each elastic and reactive collision term with a single relaxation term keeping the individual character of each elastic and reactive collision, namely,

$$
\frac{\partial f_{\alpha}}{\partial t}+c_{i}^{\alpha} \frac{\partial f_{\alpha}}{\partial x_{i}}=-\sum_{\beta=A}^{D} \zeta_{\alpha \beta}^{E}\left(f_{\alpha}-f_{\alpha \beta}^{E}\right)-\zeta_{\alpha \gamma}^{R}\left(f_{\alpha}-f_{\alpha \gamma}^{R}\right), \quad \alpha=A, \ldots, D,
$$


where the quantities $\zeta_{\alpha \beta}^{E}$ and $\zeta_{\alpha \gamma}^{R}$ denote elastic and reactive collision frequencies, related to the hard-spheres and line-of-centers cross sections, respectively. The elastic $f_{\alpha \beta}^{E}$ and the reactive $f_{\alpha \gamma}^{R}$ reference distribution functions have the Gaussian form detailed in paper [2]. They have been explicitly evaluated imposing that the production terms for mass, momentum and total energy are the same for both the approximate model equations (1) and the exact Boltzmann equation. The chosen model is capable to describe a chemical mechanism which affects the transport of masses due to the behavior of diffusion, the transport of momentum due to the behavior of shear viscosity, and the transport of energy due to thermal conductivity. In particular, all transport coefficients for this model are known in dependence on the elastic and reactive collision frequencies, mass concentrations of each constituent and temperature of the whole mixture. The model equations (1) lead to the balance equations for the number density of each constituent and to the conservation laws for momentum and total energy of the mixture which read

$$
\begin{array}{lc}
\frac{\partial n_{\alpha}}{\partial t}+\frac{\partial}{\partial x_{i}}\left(n_{\alpha} u_{i}^{\alpha}+n_{\alpha} v_{i}\right)=\tau_{\alpha}, & \frac{\partial}{\partial t}\left(\rho v_{i}\right)+\frac{\partial}{\partial x_{j}}\left(p_{i j}+\rho v_{i} v_{j}\right)=0, \\
\frac{\partial}{\partial t}\left[\frac{3}{2} n k T+\sum_{\alpha=A}^{D} n_{\alpha} \varepsilon_{\alpha}+\frac{1}{2} \rho v^{2}\right]+\frac{\partial}{\partial x_{i}}\left[q_{i}+p_{i j} v_{j}+\left(\frac{3}{2} n k T+\sum_{\alpha=A}^{D} n_{\alpha} \varepsilon_{\alpha}+\frac{1}{2} \rho v^{2}\right) v_{i}\right]=0 .
\end{array}
$$

Above, $n_{\alpha}, u_{i}^{\alpha}$ and $\varepsilon_{\alpha}$ denote the number density, diffusion velocity and formation energy of each constituent $\alpha$, respectively, whereas symbols without indexes refer to the whole mixture. The term $\tau_{\alpha}$ on the right-hand-side of Eq. (2) 1 represents the reaction rate of the constituent $\alpha$ whose explicit expression is (see paper [2])

$$
\tau_{\alpha}=v_{\alpha} n_{\alpha}^{\mathrm{eq}} n_{\gamma}^{\mathrm{eq}} k_{\sigma}^{(0)} \frac{\mathcal{A}}{k T}, \quad \text { with } \quad \mathcal{A}=k T \ln \left(\frac{n_{A} n_{B} n_{C}^{\mathrm{eq}} n_{D}^{\mathrm{eq}}}{n_{C} n_{D} n_{A}^{\mathrm{eq}} n_{B}^{\mathrm{eq}}}\right) .
$$

In the previous expressions, $k_{\sigma}^{(0)}$ denotes the first approximation to the forward $(\sigma=1)$ and backward $(\sigma=-1)$ rate constants, and $\mathcal{A}$ denotes the chemical affinity for the forward reaction

$$
k_{1}^{(0)}=\sqrt{\frac{8 \pi k T}{m_{A B}}} \mathrm{e}^{-\varepsilon_{1}^{\star}}\left(s_{1} d_{A B}\right)^{2}, \quad k_{-1}^{(0)}=\frac{n_{A}^{\mathrm{eq}} n_{B}^{\mathrm{eq}}}{n_{C}^{\mathrm{eq}} n_{D}^{\mathrm{eq}}} k_{1}^{(0)}, \quad m_{A B}=\frac{m_{A} m_{B}}{m_{A}+m_{B}}, \quad \varepsilon_{\sigma}^{\star}=\frac{\varepsilon_{\sigma}}{k T},
$$

where $s_{1}$ is the steric factor, $d_{A B}$ a mean diameter of the colliding spheres, and $\varepsilon_{\sigma}$ the activation energy of the forward and backward reaction. The closure of the above system at the Navier-Stokes, Fourier and Fick level is assured by the constitutive equations. They relate the transport fluxes $u_{i}^{\alpha}, p_{i j}, q_{i}$ to the basic fields of constituent number densities $n_{\alpha}$, mixture velocity $v_{i}$ and temperature $T$. These relations express the link between the constitutive quantities and the thermodynamical forces through the transport coefficients of diffusion $D_{\alpha \beta}$, shear viscosity $\mu$ and thermal conductivity $\lambda$. The constitutive equations can be written in the form

$$
\begin{array}{ll}
-\sum_{\beta=A}^{D} \frac{x_{\alpha}^{\mathrm{eq}} x_{\beta}^{\mathrm{eq}}}{D_{\alpha \beta}}\left(u_{i}^{\alpha}-u_{i}^{\beta}\right)=\frac{1}{p}\left(\frac{\partial p_{\alpha}}{\partial x_{i}}-\frac{\rho_{\alpha}}{\rho} \frac{\partial p}{\partial x_{i}}\right), & \text { with } \quad x_{\alpha}^{\mathrm{eq}}=\frac{n_{\alpha}^{\mathrm{eq}}}{n} \\
p_{i j}=p \delta_{i j}-\mu\left(\frac{\partial v_{i}}{\partial x_{j}}+\frac{\partial v_{j}}{\partial x_{i}}-\frac{2}{3} \frac{\partial v_{r}}{\partial x_{r}} \delta i j\right), & q_{i}=-\lambda \frac{\partial T}{\partial x_{i}}+\sum_{\alpha=A}^{D}\left(\frac{5}{2} k T+\varepsilon_{\alpha}\right) n_{\alpha}^{\mathrm{eq}} u_{i}^{\alpha} .
\end{array}
$$

In particular, observe that the Fick law (6) can be rewritten in the explicit form

$$
u_{i}^{\alpha}=-\frac{1}{p} \sum_{\beta=A}^{C}\left(\frac{\partial p_{\beta}}{\partial x_{i}}-\frac{\rho_{\beta}}{\rho} \frac{\partial p}{\partial x_{i}}\right) \Delta_{\alpha \beta}, \quad \alpha=A, B, C, \quad \text { with } \quad \sum_{\alpha=A}^{D} \rho_{\alpha} u_{i}^{\alpha}=0
$$

where $\Delta_{\alpha \beta}$ are related to the diffusion coefficients. The closed system of the reactive field equations (2)-(3) and constitutive equations (6)-(7) will now be linearized around an equilibrium state of the mixture, characterized by constant number densities $n_{\alpha}^{\mathrm{eq}}$, mixture temperature $T_{0}$ and vanishing mean velocity, through the expansions of the basic fields

$$
n_{\alpha}=n_{\alpha}^{\mathrm{eq}}+\widetilde{n}_{\alpha}, \quad v_{i}=\widetilde{v}_{i}, \quad T=T_{0}+\widetilde{T} .
$$

Above, $\widetilde{n}_{\alpha}, \widetilde{v}$ and $\widetilde{T}$ represent small unknown perturbations of the corresponding basic fields. By introducing the expansions (9) together with the constitutive equations (6)-(8) into the balance equations (2)-(3), one obtains the 
following linearized hydrodynamic system, with $n_{0}, \rho_{0}$ denoting equilibrium quantities for the whole mixture and $\widetilde{n}=\sum_{\alpha=A}^{D} \tilde{n}_{\alpha}$ :

$$
\begin{aligned}
& \frac{\partial \widetilde{n}_{A}}{\partial t}+n_{0} x_{A}^{\mathrm{eq}} \frac{\partial \widetilde{v}_{i}}{\partial x_{i}}-n_{0} x_{A}^{\mathrm{eq}} \sum_{\beta=A}^{C} \triangle_{A \beta}\left[\frac{x_{\beta}^{\mathrm{eq}}}{T_{0}}\left(1-\frac{m_{\beta} n_{0}}{\rho_{0}}\right) \frac{\partial^{2} \widetilde{T}}{\partial x_{i}^{2}}+\frac{1}{n_{0}} \frac{\partial^{2} \widetilde{n}_{\beta}}{\partial x_{i}^{2}}-\frac{m_{\beta} x_{\beta}^{\mathrm{eq}}}{\rho_{0}} \frac{\partial^{2} \widetilde{n}}{\partial x_{i}^{2}}\right] \\
& +n_{0} x_{A}^{\mathrm{eq}} x_{B}^{\mathrm{eq}} k_{1}^{(0)}\left[\frac{\widetilde{n}_{A}}{x_{A}^{\mathrm{eq}}}+\frac{\widetilde{n}_{B}}{x_{B}^{\mathrm{eq}}}-\frac{\widetilde{n}_{C}}{x_{C}^{\mathrm{eq}}}-\frac{\widetilde{n}_{D}}{x_{D}^{\mathrm{eq}}}\right]=0, \\
& \frac{\partial \widetilde{n}_{B}}{\partial t}+n_{0} x_{B}^{\mathrm{eq}} \frac{\partial \widetilde{v}_{i}}{\partial x_{i}}-n_{0} x_{B}^{\mathrm{eq}} \sum_{\beta=A}^{C} \triangle_{B \beta}\left[\frac{x_{\beta}^{\mathrm{eq}}}{T_{0}}\left(1-\frac{m_{\beta} n_{0}}{\rho_{0}}\right) \frac{\partial^{2} \widetilde{T}}{\partial x_{i}^{2}}+\frac{1}{n_{0}} \frac{\partial^{2} \widetilde{n}_{\beta}}{\partial x_{i}^{2}}-\frac{m_{\beta} x_{\beta}^{\mathrm{eq}}}{\rho_{0}} \frac{\partial^{2} \widetilde{n}}{\partial x_{i}^{2}}\right] \\
& +n_{0} x_{A}^{\mathrm{eq}} x_{B}^{\mathrm{eq}} k_{1}^{(0)}\left[\frac{\widetilde{n}_{A}}{x_{A}^{\mathrm{eq}}}+\frac{\widetilde{n}_{B}}{x_{B}^{\mathrm{eq}}}-\frac{\widetilde{n}_{C}}{x_{C}^{\mathrm{eq}}}-\frac{\widetilde{n}_{D}}{x_{D}^{\mathrm{eq}}}\right]=0, \\
& \frac{\partial \widetilde{n}_{C}}{\partial t}+n_{0} x_{C}^{\mathrm{eq}} \frac{\partial \widetilde{v}_{i}}{\partial x_{i}}-n_{0} x_{C}^{\mathrm{eq}} \sum_{\beta=A}^{C} \triangle_{C \beta}\left[\frac{x_{\beta}^{\mathrm{eq}}}{T_{0}}\left(1-\frac{m_{\beta} n_{0}}{\rho_{0}}\right) \frac{\partial^{2} \widetilde{T}}{\partial x_{i}^{2}}+\frac{1}{n_{0}} \frac{\partial^{2} \widetilde{n}_{\beta}}{\partial x_{i}^{2}}-\frac{m_{\beta} x_{\beta}^{\mathrm{eq}}}{\rho_{0}} \frac{\partial^{2} \widetilde{n}}{\partial x_{i}^{2}}\right] \\
& -n_{0} x_{A}^{\mathrm{eq}} x_{B}^{\mathrm{eq}} k_{1}^{(0)}\left[\frac{\tilde{n}_{A}}{x_{A}^{\mathrm{eq}}}+\frac{\widetilde{n}_{B}}{x_{B}^{\mathrm{eq}}}-\frac{\widetilde{n}_{C}}{x_{C}^{\mathrm{eq}}}-\frac{\tilde{n}_{D}}{x_{D}^{\mathrm{eq}}}\right]=0, \\
& \frac{\partial \widetilde{n}_{D}}{\partial t}+n_{0} x_{D}^{\mathrm{eq}} \frac{\partial \widetilde{v}_{i}}{\partial x_{i}}+n_{0} \sum_{\alpha=A}^{C} \sum_{\beta=A}^{C} x_{\alpha}^{\mathrm{eq}} \frac{m_{\alpha}}{m_{D}} \triangle_{\alpha \beta}\left[\frac{x_{\beta}^{\mathrm{eq}}}{T_{0}}\left(1-\frac{m_{\beta} n_{0}}{\rho_{0}}\right) \frac{\partial^{2} \widetilde{T}}{\partial x_{i}^{2}}+\frac{1}{n_{0}} \frac{\partial^{2} \widetilde{n}_{\beta}}{\partial x_{i}^{2}}-\frac{m_{\beta} x_{\beta}^{\mathrm{eq}}}{\rho_{0}} \frac{\partial^{2} \tilde{n}}{\partial x_{i}^{2}}\right] \\
& -n_{0} x_{A}^{\mathrm{eq}} x_{B}^{\mathrm{eq}} k_{1}^{(0)}\left[\frac{\widetilde{n}_{A}}{x_{A}^{\mathrm{eq}}}+\frac{\widetilde{n}_{B}}{x_{B}^{\mathrm{eq}}}-\frac{\widetilde{n}_{C}}{x_{C}^{\mathrm{eq}}}-\frac{\widetilde{n}_{D}}{x_{D}^{\mathrm{eq}}}\right]=0, \\
& \frac{\partial \widetilde{v}_{i}}{\partial t}+\frac{k n_{0}}{\rho_{0}} \frac{\partial \widetilde{T}}{\partial x_{i}}+\sum_{\alpha=A}^{D} \frac{k T_{0}}{\rho_{0}} \frac{\partial \widetilde{n}_{\alpha}}{\partial x_{i}}-\frac{\mu}{\rho_{0}}\left[\frac{\partial^{2} \widetilde{v}_{i}}{\partial x_{1}^{2}}+\frac{\partial^{2} \widetilde{v}_{i}}{\partial x_{2}^{2}}+\frac{\partial^{2} \widetilde{v}_{i}}{\partial x_{3}^{2}}\right] \\
& -\frac{1}{3} \frac{\mu}{\rho_{0}}\left[\frac{\partial^{2} \widetilde{v}_{1}}{\partial x_{1} \partial x_{i}}+\frac{\partial^{2} \widetilde{v}_{2}}{\partial x_{2} \partial x_{i}}+\frac{\partial^{2} \widetilde{v}_{3}}{\partial x_{3} \partial x_{i}}\right]=0 \text {, } \\
& \frac{\partial \widetilde{T}}{\partial t}+\frac{2}{3} T_{0} \frac{\partial \widetilde{v}_{i}}{\partial x_{i}}-\frac{2}{3} \frac{\lambda}{k n_{0}} \frac{\partial^{2} \widetilde{T}}{\partial x_{i}^{2}}+\frac{2}{3} E^{*} x_{A}^{\mathrm{eq}} x_{B}^{\mathrm{eq}} T_{0} k_{1}^{(0)}\left[\frac{\widetilde{n}_{A}}{x_{A}^{\mathrm{eq}}}+\frac{\widetilde{n}_{B}}{x_{B}^{\mathrm{eq}}}-\frac{\widetilde{n}_{C}}{x_{C}^{\mathrm{eq}}}-\frac{\widetilde{n}_{D}}{x_{D}^{\mathrm{eq}}}\right] \\
& -\frac{2}{3} T_{0} \sum_{\alpha=A}^{C} \sum_{\beta=A}^{C} \triangle_{\alpha \beta} \frac{m_{D}-m_{\alpha}}{m_{D}}\left[\frac{1}{T_{0}} x_{\alpha}^{\mathrm{eq}} x_{\beta}^{\mathrm{eq}}\left(1-\frac{n_{0} m_{\beta}}{\rho_{0}}\right) \frac{\partial^{2} \widetilde{T}}{\partial x_{i}^{2}}+\frac{x_{\alpha}^{\mathrm{eq}}}{n_{0}} \frac{\partial^{2} \widetilde{n}_{\beta}}{\partial x_{i}^{2}}-\frac{m_{\beta}}{\rho_{0}} x_{\alpha}^{\mathrm{eq}} x_{\beta}^{\mathrm{eq}} \frac{\partial^{2} \widetilde{n}}{\partial x_{i}^{2}}\right]=0 .
\end{aligned}
$$

\section{HYDRODYNAMIC APPROACH FOR LIGHT SCATTERING PROBLEM}

To study light scattering phenomenon in the considered reactive mixture, one can start from the hydrodynamic equations in the approximate linear theory (10)-(15) to describe the response of the equilibrium reactive system to the incident light field. The deviations of the light field due to its interaction with the system can be examined in terms of the time-correlation functions of the partial number densities fluctuations. Such correlation functions provide a suitable tool to evaluate the intensity of the light scattered by the reactive mixture. The knowledge of auto and cross correlation functions of the partial number densities leads to the evaluation of the spectrum of number density fluctuations with scattering vector $\mathbf{q}$, which is also known in literature as the dynamic structure factor $S(\mathbf{q}, \omega)$. Its definition [4] is given in terms of the auto correlation function of the spatial Fourier transform of the dielectric constant fluctuation $\delta \varepsilon(\mathbf{x}, t)$. Since such correlation is a real and even time-reversal function, the dynamic structure factor $S(\mathbf{q}, \omega)$ is explicitly given by

$$
S(\mathbf{q}, \omega)=\operatorname{Re}[\langle\delta \varepsilon(\mathbf{q}, i \omega) \delta \varepsilon(\mathbf{q}, 0)\rangle]
$$

where $\omega$ is the shift in the angular frequency in the scattering process, and

$$
\langle\delta \varepsilon(\mathbf{q}, i \omega) \delta \varepsilon(\mathbf{q}, 0)\rangle=\int_{0}^{\infty} e^{-i \omega t} \iint\left\langle\delta \varepsilon\left(\mathbf{x}^{\prime}, t\right) \delta \varepsilon(\mathbf{x}, 0)\right\rangle e^{i \mathbf{q} \cdot\left(\mathbf{x}^{\prime}-\mathbf{x}\right)} d \mathbf{x} d \mathbf{x}^{\prime} d t
$$


is the Laplace-Fourier transform of the ensemble average of the dielectric fluctuation over the initial state of the mixture. For the considered quaternary reactive mixture, the dielectric fluctuation $\delta \varepsilon(\mathbf{x}, t)$ is a linear combination of the constituent number density fluctuations whose coefficients $a_{\alpha}$ denote the constituent's atomic polarizabilities, namely

$$
\delta \varepsilon(\mathbf{x}, t)=\sum_{\alpha=A}^{D} a_{\alpha} \delta n_{\alpha}(\mathbf{x}, t) .
$$

Equation (18) extends the Clausius-Mossotti equation to the considered dilute gas mixture (see the book [5]). Consequently, from Eqs. (16) and (18), the dynamic structure factor can be expressed in terms of the Laplace-Fourier transforms of the cross and auto correlation functions of the constituent number density fluctuations, that is

$$
S(\mathbf{q}, \omega)=\sum_{\alpha=A}^{D} \sum_{\gamma=A}^{D} a_{\alpha} a_{\gamma} \operatorname{Re}\left[\left\langle\delta n_{\alpha}(\mathbf{q}, i \omega) \delta n_{\gamma}(\mathbf{q}, 0)\right\rangle\right] .
$$

By invoking the Onsager reciprocity regression hypothesis, which states that the spontaneous microscopic fluctuations regress to equilibrium according to the same hydrodynamic laws which govern the relaxation to equilibrium of the macroscopic perturbations, the dynamic structure factor assumes the equivalent form

$$
S(\mathbf{q}, \omega)=\sum_{\alpha=A}^{D} \sum_{\gamma=A}^{D} a_{\alpha} a_{\gamma} \operatorname{Re}\left[\left\langle\widetilde{n}_{\alpha}(\mathbf{q}, i \omega) \widetilde{n}_{\gamma}(\mathbf{q}, 0)\right\rangle\right]
$$

In order to determine the correlation functions $\left\langle\widetilde{n}_{\alpha}(\mathbf{q}, i \omega) \widetilde{n}_{\gamma}(\mathbf{q}, 0)\right\rangle$, first write the Fourier-Laplace transform of the linearized hydrodynamic equations (10)-(15) in the following matrix form:

$$
(i \omega \mathbf{I}+\mathbf{M}(\mathbf{q})) \Psi(\mathbf{q}, i \omega)=\Psi(\mathbf{q}, 0),
$$

where $\mathbf{I}$ is the identity matrix and

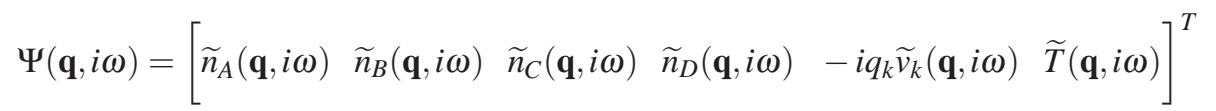

is the vector of the Fourier-Laplace transforms of the field perturbations. Moreover, the elements of the so-called hydrodynamic matrix $\mathbf{M}(\mathbf{q})$, besides the system parameters, depend on the equilibrium state and transport coefficients. Their explicit expressions will be provided in the extended paper [6]. Next, the algebraic system (21) will be solved for $\widetilde{n}_{\alpha}(\mathbf{q}, i \omega)$. Multiplying the resulting equation by $\widetilde{n}_{\gamma}(\mathbf{q}, 0)$ and performing the ensemble average over the initial states of the system, one gets

$$
\left\langle\widetilde{n}_{\alpha}(\mathbf{q}, i \omega) \widetilde{n}_{\gamma}(\mathbf{q}, 0)\right\rangle=(i \omega \mathbf{I}+\mathbf{M}(\mathbf{q}))_{\alpha \gamma}^{-1}\left\langle\left|\widetilde{n}_{\gamma}(\mathbf{q}, 0)\right|^{2}\right\rangle
$$

where the equal-time correlation function $\left\langle\left|\widetilde{n}_{\gamma}(\mathbf{q}, 0)\right|^{2}\right\rangle$ is known from the assigned initial conditions. Finally, the dynamic structure factor can be evaluated as

$$
S(\mathbf{q}, \omega)=\sum_{\alpha=A}^{D} \sum_{\gamma=A}^{D} a_{\alpha} a_{\gamma} \operatorname{Re}\left[(i \omega \mathbf{I}+\mathbf{M}(\mathbf{q}))_{\alpha \gamma}^{-1}\right]\left\langle\left|\widetilde{n}_{\gamma}(\mathbf{q}, 0)\right|^{2}\right\rangle
$$

\section{LIGHT SCATTERING SPECTRUM}

The theoretical analysis of the previous section is here applied to two different mixtures of the Hydrogen-Chlorine system where the elementary bimolecular chemical reaction $\mathrm{H}_{2}+\mathrm{Cl} \rightleftharpoons \mathrm{HCl}+\mathrm{H}$ occurs. The considered mixtures have different equilibrium constituent concentrations, the same equilibrium temperature $T_{0}=1500 \mathrm{~K}$ and positive value of the chemical affinity $\mathcal{A}$ which corresponds to an exothermic reaction. Accordingly, two cases are investigated, namely: (a) $x_{A}^{\mathrm{eq}}=x_{B}^{\mathrm{eq}}=0.33$ and $x_{C}^{\mathrm{eq}}=x_{D}^{\mathrm{eq}}=0.17$; (b) $x_{A}^{\mathrm{eq}}=0.1, x_{B}^{\mathrm{eq}}=0.618, x_{C}^{\mathrm{eq}}=0.082$ and $x_{D}^{\mathrm{eq}}=0.2$. In case (a) the concentrations $x_{A}^{\mathrm{eq}}, x_{B}^{\mathrm{eq}}, x_{C}^{\mathrm{eq}}$ and $x_{D}^{\mathrm{eq}}$ of the constituents $\mathrm{H}_{2}, \mathrm{Cl}, \mathrm{HCl}$ and $\mathrm{H}$, respectively, were assumed in such a way that $x_{A}^{\mathrm{eq}}=x_{B}^{\mathrm{eq}}$ and $x_{C}^{\mathrm{eq}}=x_{D}^{\mathrm{eq}}$. Moreover $x_{A}^{\mathrm{eq}}$ and $x_{B}^{\mathrm{eq}}$ were chosen and $x_{C}^{\mathrm{eq}}$ and $x_{D}^{\mathrm{eq}}$ were obtained from the constraint $x_{A}^{\mathrm{eq}}+x_{B}^{\mathrm{eq}}+x_{C}^{\mathrm{eq}}+x_{D}^{\mathrm{eq}}=1$ and from the mass action law. In case (b) the concentrations $x_{A}^{\mathrm{eq}}$ and $x_{D}^{\mathrm{eq}}$ were chosen, and $x_{B}^{\mathrm{eq}}$ 
TABLE 1. Partial uniformity parameters $(y=15)$.

\begin{tabular}{ccccc}
\hline & $y_{A}$ & $y_{B}$ & $y_{C}$ & $y_{D}$ \\
\hline case (a) & 10.062 & 16.449 & 29.255 & 5.081 \\
\hline case (b) & 7.165 & 9.551 & 18.455 & 3.415 \\
\hline
\end{tabular}

and $x_{C}^{\mathrm{eq}}$ were also obtained in the same manner as before. The values of the transport coefficients, activation energy, Arrhenius parameter and steric factor are those of paper [2] for the mixture of case (a) and those of paper [7] for the mixture of case (b).

In Figures 1 and 2 the light scattering spectrum is represented as function of the reduced angular frequency $\omega / c q$ for different values of the uniformity parameter $y=(\tau c q)^{-1}$, with $\tau$ an effective relaxation time, $c$ the adiabatic sound speed of the mixture and $q$ the intensity of the scattering vector. Since $y$ is proportional to the ratio between the wavelength of the incident light and the effective mean free path $\tau c$, the hydrodynamic equations (10)-(15) can be used to describe light scattering spectrum if the condition $y \gg 1$ holds. In this case, the light scattering spectrum has the well-known triplet shape consisting of a central Rayleigh peak and two Brillouin peaks that are shifted symmetrically about the origin. The widths of the Rayleigh and Brillouin lines depend on the transport coefficients. The Brillouin shifts are proportional to the adiabatic sound velocity of the mixture, so that the position of the Brillouin peaks moves outwards of the center of the spectrum when the adiabatic sound velocity of the mixture increases. Furthermore, in the kinetic regime $(y \sim 1)$ the central Rayleigh and lateral Brillouin peaks tend to broaden whereas, in the hydrodynamic regime $(y \gg 1)$ the intensity of the peaks grows and they become narrower. These features can be recognized in Figures 1 and 2 for the selected range of the uniformity parameter $y$. In addition, the results shown in these figures allow to appreciate the effects of the chemical reaction, since the mixtures of cases (a) and (b) have been considered in the reactive case (solid line) and also when the chemical reaction is absent (dashed line). In both pictures, the effect of the chemical reaction produces higher Rayleigh and lower Brillouin peaks. In particular, the explanation for the behavior of the Rayleigh and Brillouin peaks is based on the fact that their widths are proportional to the transport coefficients and these last are smaller for reactive mixtures than for inert ones, see paper [2].

To understand better the curves in the figures one defines a partial uniformity parameter as

$$
y_{\alpha}=\frac{p_{\alpha}}{\mu_{\alpha} q v_{\alpha}}, \quad \text { where } \quad v_{\alpha}=\sqrt{\frac{5 k T_{0}}{3 m_{\alpha}}}, \quad p_{\alpha}=k n_{\alpha}^{\mathrm{eq}} T_{0},
$$

with $\mu_{\alpha}$ being the shear viscosity of constituent $\alpha$ in the mixture. The values of the partial uniformity parameters for $y=15$ are given in Table 1 and the partial polarizabilities satisfy the conditions $\alpha_{A} \approx \alpha_{D} \approx \alpha_{B} / 3 \approx \alpha_{C} / 3$. Since the polarizabilities of $\mathrm{Cl}$ and $\mathrm{HCl}$ are almost three times larger than the polarizabilities of $\mathrm{H}_{2}$ and $\mathrm{H}$, the light scattering spectra showed in Figure 1 and 2 are basically determined by these constituents. According to the values of the partial uniformity parameters given in Table 1, it can be verified for case (a) that both $\mathrm{Cl}$ and $\mathrm{HCl}$ are in the hydrodynamic regime since condition $y_{\alpha} \gg 1$ is satisfied. However, for case (b) one verifies that $\mathrm{HCl}$ satisfies the hydrodynamic condition, but $\mathrm{Cl}$ does not meet this condition. This explains why the Rayleigh and Brillouin peaks are less evident in case (b) than in case (a).

One may also obtain that the effect of the chemical reaction on the Rayleigh and Brillouin lines are less pronounced by decreasing the temperature of the mixture. Lastly, it is important to mention that the light scattering spectrum of gaseous mixtures is very sensitive to the presence of thermal-diffusion effects, in particular, when the molecular masses of constituents are very distinct. Note that the considered hydrodynamic model does not take into account thermal-diffusion effects, so that an improvement in the light scattering predictions can be obtained if these effects are introduced into the model. The introduction of these effects into the hydrodynamic model and their influence on light scattering of a chemically reacting gas mixture will be the subject of a forthcoming paper [6].

\section{ACKNOWLEDGMENTS}

The paper is partially supported by Brazilian Research Council (CNPq), by Minho University Mathematics Centre (CMAT-FCT) and Project FCT-PTDC/MAT/68615/2006, and by Italian National Project GNFM 2009/10. 


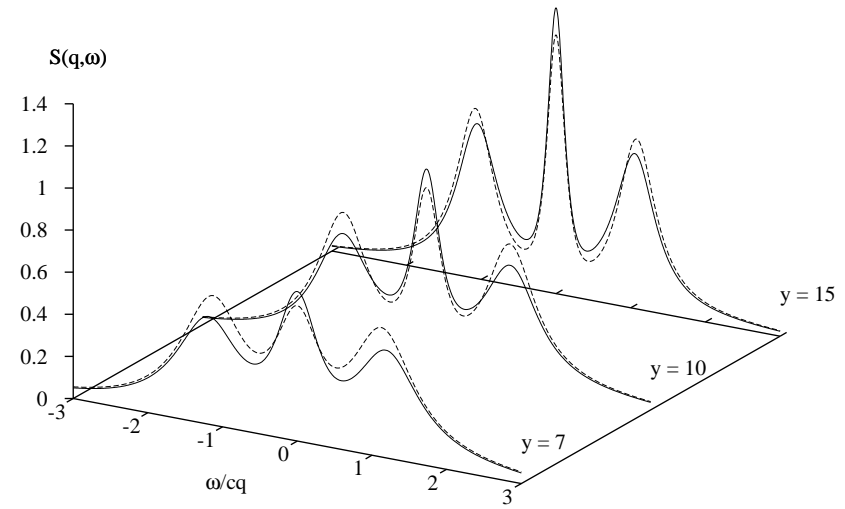

FIGURE 1. Light scattering spectra as functions of the reduced angular frequency $\omega / c q$ for different values of the uniformity parameter $y$. Case (a) $x_{A}=x_{B}=0.33, x_{C}=x_{D}=0.17$. Reactive mixture (solid line) and inert mixture (dashed line).

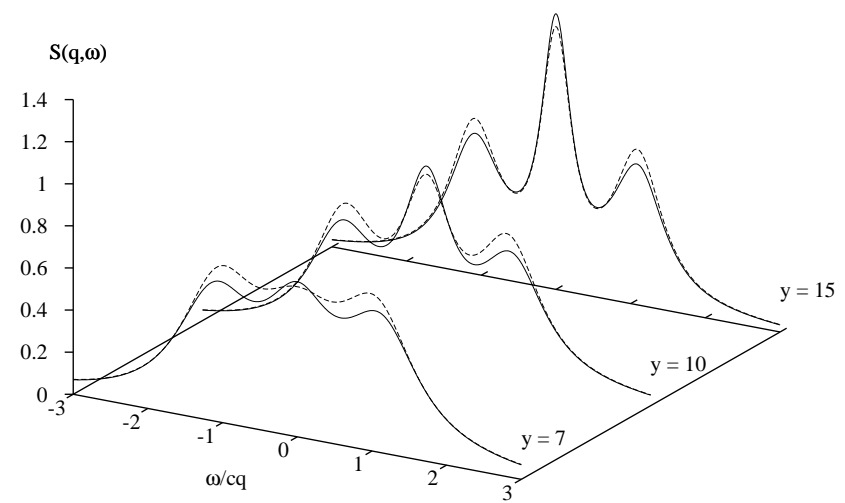

FIGURE 2. Light scattering spectra as functions of the reduced angular frequency $\omega / c q$ for different values of the uniformity parameter $y$. Case (b): $x_{A}=0.1, x_{B}=0.618, x_{C}=0.082, x_{D}=0.2$. Reactive mixture (solid line) and inert mixture (dashed line).

\section{REFERENCES}

1. W. Weiss, I. Muller, Continuum Mech. Thermodyn. 7, 123-177 (1995).

2. G. M. Kremer, M. Pandolfi Bianchi, A. J. Soares, Phys. Fluids, 18, 037104 (2006).

3. W. Marques Jr., G. M. Alves, G. M. Kremer, Physica A, 323, 401-412 (2003).

4. B. Berne, R. Pecora, Dynamic Light Scattering, Wiley, New York, 1976.

5. M. Born, E. Wolf, Principles of Optics, Cambridge University Press, Cambridge, 1999.

6. W. Marques Jr., G. M. Kremer, M. Pandolfi Bianchi, A. J. Soares, paper in preparation.

7. G. M. Kremer, M. Pandolfi Bianchi, A. J. Soares, Il Nuovo Cimento, 33, 103-110 (2010). 